\title{
S51-01
}

\section{SUBJECTIVE WELL-BEING UNDER NEUROLEPTIC TREATMENT. RELEVANCE FOR COMPLIANCE AND REMISSION}

\section{Naber}

Psychiatry, University Medical Center Hamburg-Eppendorf, Hamburg, Germany

Within the last decade, success criteria of antipsychotic treatment became more ambitious. The most important change is the long overdue consideration of the patient's perspective. Among other scales, a self-report instrument has been constructed to evaluate "subjective well-being under neuroleptics" (SWN). Studies indicate:

a. schizophrenic patients, if no longer acutely psychotic or suffering from severe cognitive deficits, are able to reliably assess their SWN,

b. high SWN is correlated with high compliance,

c. atypical antipsychotics increase SWN

d. individual improvements of SWN and of PANSS are not strongly related ( $r=-.30--.40$ ), and e) dopamine D2 receptor blockade is highly correlated to reduced SWN $(r=.66-.76)$.

Several open trials reveal the relevance of early improvement of subjective well-being: In a 12-week trial with 727 patients, $95 \%$ of those with early subjective response (within 4 weeks) showed later subjective and/or psychopathological improvement, but only $9 \%$ without early subjective response showed later improvement. In another 3-year trial of 2690 patients, again psychopathological response as well as symptomatic and functional remission were mostly related to early (within first 3 months) subjective improvement. Finally, in a first-episode study of 110 patients with a follow-up of 5 years, within the first 6 weeks of antipsychotic treatment only improvement of SWN was related to enduring symptomatic remission $(p=.004)$ while early reduction of PANSS did not predict long-term course. These data indicate the usefulness of self-rating in schizophrenia, insufficient subjective improvement needs to be identified early. 